\title{
Disruption of D2R $\times$ DISC1 binding prevents conditioned place preference induced by morphine in mice
}

\author{
M.V. Starostina ${ }^{1 *}$, N.S. Sorokina ${ }^{1}$ T.V. Lipina ${ }^{2,3}$ \\ ${ }^{1}$ Institute of Molecular Biology Biophysics, Novosibirsk, Russia \\ ${ }^{2}$ Scientific Research Institute of Physiology and Basic Medicine, Novosibirsk, Russia \\ ${ }^{3}$ Novosibirsk State University, Novosibirsk, Russia \\ *e-mail:marina@niimbb.ru
}

Key words: conditioned place preference, morphine, dopamine receptor, DISC1

Motivation and Aim: Dopaminergic system plays an important role in the development of drug dependence. DISC1 (Disrupted-In-Schizophrenia-1) is a scaffold protein that interacts with numerous proteins in various cellular compartments. DISC1 is involved in the regulation of functions of dopaminergic system in brain [1]. Recently, direct binding of DISC1 to a type 2 dopamine receptor (D2R) has been shown, leading to inhibition of receptor internalization, which resulted in hyperactivation of dopaminergic system [2]. A peptide analogue of D2R binding site was synthesized, and a conjugate of this peptide with a peptide fragment from immunodeficiency virus envelope (TATD2pep) competitively inhibit D2 receptor interaction with DISC1 when administered systemically [2]. The aim of our work was to probe the effect of TAT-D2pep on the formation of conditioned place preference (CPP) in mice.

Methods and Algorithms: CPP to morphine was produced in C57BL/6J mice with an unbiased CPP procedure employing a three-compartment apparatus. The mice were divided into 4 groups. During the conditioning session, control animals received injections of saline (Sal), whereas experimental groups were treated by morphine/Sal, TAT-D2pep/Sal or TAT-D2pep injected in 15 minutes before morphine/Sal.

Results: Mice treated by TAT-D2pep/Sal did not differ from the control group in CPP, showing no preference to the compartment associated with TAT-D2pep, i. e. the peptide did not affect rewarding processes in mice. Mice treated by morphine/Sal spent significantly more time in the compartment associated with morphine, supporting the formation of CPP. Administration of morphine after the extinction session, recovered CPP in this experimental group of mice. Injection of TAT-D2pep prior to the administration of morphine, significantly reduced the time spent in morphine-associated compartment than in animals receiving only morphine and was not differ from the control group.

Conclusion: The peptide, which prevents the binding of D2R to the DISC1 protein, disrupted the CPP formation, induced by morphine. These data suggest the involvement of DISC1 in the development of opiate dependence.

\section{References}

1. Dahoun T. et al. (2017) The impact of Disrupted-in-Schizophrenia 1 (DISC1) on the dopaminergic system: a systematic review. Transl Psychiatry. 7:e1015; DOI 10.1038/tp.2016.282.

2. Su P. et al. (2014) A dopamine D2 receptor-DISC1 protein complex may contribute to antipsychotic-like effects. Neuron. 84(6):1302-1316. 